\title{
Stratorotational instability in MHD Taylor-Couette flows
}

\author{
G. Rüdiger ${ }^{1}$ and D. A. Shalybkov ${ }^{2}$ \\ 1 Astrophysikalisches Institut Potsdam, An der Sternwarte 16, 14482 Potsdam, Germany \\ e-mail: gruediger@aip.de \\ 2 A.F. Ioffe Institute for Physics and Technology, 194021, St. Petersburg, Russia \\ Received 12 February 2008 / Accepted 10 September 2008
}

\section{ABSTRACT}

\begin{abstract}
Aims. The stability of the dissipative Taylor-Couette flow with a stable axial density stratification and a prescribed azimuthal magnetic field is considered.

Methods. Global nonaxisymmetric solutions of the linearized MHD equations with toroidal magnetic field, density stratification, and differential rotation are found for both insulating and conducting cylinders.

Results. Hydrodynamic calculations for various gap widths show that flat rotation laws such as the Kepler rotation are always unstable against SRI. Quasigalactic rotation laws, however, are stable for wide gaps. The influence of a current-free toroidal magnetic field on SRI strongly depends on the magnetic Prandtl number Pm: SRI is supported by Pm $>1$ and it is suppressed by Pm $\lesssim 1$. For rotation laws that are too flat, when the hydrodynamic SRI ceases, a smooth transition exists to the instability that the toroidal magnetic field produces in combination with the differential rotation. For the first time this nonaxisymmetric azimuthal magnetorotational instability (AMRI) has been computed in the presence of an axial density gradient.

If the magnetic field between the cylinders is not current-free, then the Tayler instability occurs, too. The transition from the nonmagnetic centrifugal instability to the magnetic Tayler instability in the presence of differential rotation and density stratification proves to be complex. Most spectacular is the "ballooning" of the stability domain by the density stratification: already a small rotation stabilizes magnetic fields against the Tayler instability.

An azimuthal component of the electromotive force $\left\langle\boldsymbol{u}^{\prime} \times \boldsymbol{B}^{\prime}\right\rangle$ for the instability only exists for density-stratified flows. The related $\alpha$-effect for magnetic-influenced SRI with Kepler rotation appears to be positive for negative $\mathrm{d} \rho / \mathrm{d} z<0$.
\end{abstract}

Key words. accretion, accretion disks - turbulence - instabilities - magnetohydrodynamics (MHD)

\section{Introduction}

This work is motivated by the theoretical and experimental progress in studies of the stratorotational instability (SRI) and the magnetorotational instability (MRI) in MHD Taylor-Couette experiments. It has been shown theoretically (Molemaker et al. 2001; Yavneh et al. 2001; Dubrulle et al. 2005; Shalybkov \& Rüdiger 2005; Umurhan 2006) and in the laboratory (Le Bars \& Le Gal 2007) that a combination of a centrifugal-stable nonuniform rotation law and a stable axial density stratification leads to the SRI in the Taylor-Couette flow. This instability exists only for nonaxisymmetric disturbances. On the other hand, there are also nonaxisymmetric instabilities for a combination of Rayleigh-stable rotation laws and azimuthal magnetic fields (Pitts \& Tayler 1985). The question is whether the combination of density stratification, differential rotation, and toroidal fields acts as stabilizing or destabilizing or whether even new instabilities arise.

Such a combination of axial density stratification, stable rotation law, and strong toroidal magnetic field is the typical constellation in accretion disks (Ogilvie \& Pringle 1996; Curry \& Pudritz 1996; Papaloizou \& Terquem 1997). There the rotation is Keplerian with $\Omega \propto R^{-3 / 2}$ and the toroidal field is generated from weak large-scale poloidal fields by induction due to the differential rotation. The standard case is that the resulting toroidal field strongly exceeds the amplitude of the original poloidal field if the magnetic Reynolds number of the differential rotation $\mathrm{Rm}=\Omega R^{2} / \eta$ is much larger than unity. If this is true, then the standard MRI, i.e. the influence of the poloidal field on the stability of the differential rotation, would be of minor importance $^{1}$. The question is whether the toroidal magnetic field can reach such high amplitudes or whether it already becomes unstable for much lower values. It is known that toroidal fields with strong electric currents become unstable (Tayler instability, "TI") but it is also known that, in combination with differential rotation, toroidal fields also become unstable, which are current-free in the considered domain (azimuthal MRI, "AMRI", see Rüdiger et al. 2007a). In the latter case, with $B_{\phi} \propto R^{-1}$, the questions are whether the density stratification destabilizes AMRI and/or whether the toroidal field stabilizes the SRI too strongly, so that its existence becomes basically suppressed.

We consider the interaction of the differential rotation with both an axial density stratification and a toroidal magnetic field in the simplified Taylor-Couette geometry. The density stratification is always supposed to be stable, but the magnetic field and the rotation law between the cylinders can be both stable or unstable. If, in particular, the toroidal field is Taylerunstable, then the interaction of differential rotation, density stratification and magnetic field becomes very complex. We find stabilization and destabilization to depend strongly on the magnetic Prandtl number. Again, in experiments, for small magnetic Prandtl number, the flows are predicted to be stabilized. For galaxies and protoneutron stars (PNS, with their high magnetic Prandtl numbers, we find the opposite. The magnetic influence supports the SRI leading to even smaller Reynolds numbers than in hydrodynamics.

\footnotetext{
1 The same is true for calculations about the stability of strong poloidal fields without or with rotation.
} 


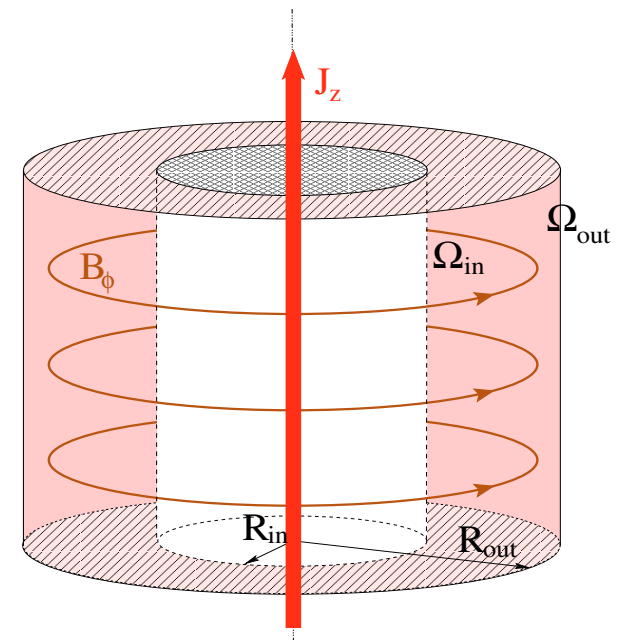

Fig. 1. The geometry of the problem - two concentric cylinders with radii $R_{\text {in }}$ and $R_{\text {out }}$ rotating with $\Omega_{\text {in }}$ and $\Omega_{\text {out }} . B_{\phi}$ is the azimuthal magnetic field which, generally, is produced by both an axial current inside the inner cylinder and an axial current through the fluid.

\section{The Taylor-Couette geometry}

A Taylor-Couette container is considered that confines a toroidal magnetic field with given amplitudes at the cylinder walls that rotate with different rotation rates $\Omega$ (see Fig. 1). The fluid between the cylinders is assumed to be incompressible and dissipative with the kinematic viscosity $v$ and the magnetic diffusivity $\eta$. Then the rotation law $\Omega(R)$ in the fluid is

$\Omega=a+\frac{b}{R^{2}}$,

with

$a=\frac{\mu_{\Omega}-\hat{\eta}^{2}}{1-\hat{\eta}^{2}} \Omega_{\mathrm{in}}, \quad b=\frac{1-\mu_{\Omega}}{1-\hat{\eta}^{2}} R_{\mathrm{in}}^{2} \Omega_{\mathrm{in}}$,

and

$\hat{\eta}=\frac{R_{\text {in }}}{R_{\text {out }}}, \quad \quad \mu_{\Omega}=\frac{\Omega_{\text {out }}}{\Omega_{\text {in }}}$.

Cylindric coordinates $(R, \phi, z)$ are used, and $\Omega_{\text {in }}$ and $\Omega_{\text {out }}$ are the imposed rotation rates at the inner and outer cylinders of radii $R_{\text {in }}$ and $R_{\text {out }}$.

Similarly, the magnetic profiles are restricted by the azimuthal component of the induction equation to

$B_{\phi}=A R+\frac{B}{R}$

where the first term corresponds to a uniform axial electric current at radius $R$ and the second term is current-free for $R>0$. In analogy with $\mu_{\Omega}$, it is useful to define the quantity

$\mu_{B}=\frac{B_{\text {out }}}{B_{\text {in }}}=\frac{A R_{\text {out }}+B R_{\text {out }}^{-1}}{A R_{\text {in }}+B R_{\text {in }}^{-1}}$,

measuring the variation in $B_{\phi}$ between the cylinders. The coefficients $A$ and $B$ are

$A=\frac{B_{\text {in }}}{R_{\text {in }}} \frac{\hat{\eta}\left(\mu_{B}-\hat{\eta}\right)}{1-\hat{\eta}^{2}}$

$$
B=B_{\text {in }} R_{\text {in }} \frac{1-\mu_{B} \hat{\eta}}{1-\hat{\eta}^{2}}
$$

The MHD equations for incompressible stratified fluids are

$$
\begin{aligned}
& \frac{\partial \boldsymbol{u}}{\partial t}+(\boldsymbol{u} \nabla) \boldsymbol{u}=-\frac{1}{\rho} \nabla P+\boldsymbol{g}+v \Delta \boldsymbol{u}+\frac{1}{\mu_{0}} \operatorname{curl} \boldsymbol{B} \times \boldsymbol{B}, \\
& \frac{\partial \boldsymbol{B}}{\partial t}=\operatorname{curl}(\boldsymbol{u} \times \boldsymbol{B})+\eta \Delta \boldsymbol{B}, \\
& \frac{\partial \rho}{\partial t}+(\boldsymbol{u} \nabla) \rho=0,
\end{aligned}
$$

and

$\operatorname{div} \boldsymbol{u}=0, \quad \operatorname{div} \boldsymbol{B}=0$,

where $\boldsymbol{u}$ is the velocity, $\boldsymbol{B}$ the magnetic field, $P$ the pressure, $g$ the gravitational acceleration (supposed as vertical and constant), $v$ the kinematic viscosity, $\eta$ the magnetic diffusivity, and $\mu_{0}$ the magnetic constant.

In the presence of a vertical density gradient $(\rho=\rho(z)$, it has been shown for $\boldsymbol{B}=0$ that the system (7) allows the angular velocity profile (1) only in the limit of slow rotation and small stratification

$$
\left|\frac{R \Omega^{2}}{g}\right| \ll 1, \quad\left|\frac{\operatorname{dlog}(\rho)}{\operatorname{dlog}(z)}\right| \ll 1 .
$$

It easy to show that the same is true of $B \neq 0$. Thus, we are interested in the stability/instability of the basic state

$$
\begin{array}{ll}
\boldsymbol{U}=(0, R \Omega(R), 0), & \boldsymbol{B}=\left(0, B_{\phi}(R), 0\right), \\
P=P_{0}(R)+P_{1}(R, z), & \rho=\rho_{0}+\rho_{1}(z),
\end{array}
$$

where $\rho_{0}$ is the uniform reference density, $P$ the total pressure including the magnetic one with $\left|P_{1} / P_{0}\right| \ll 1$, and $\left|\rho_{1} / \rho_{0}\right| \ll 1$.

The linear stability problem is considered for the perturbed state of $\boldsymbol{U}+\boldsymbol{u}, \boldsymbol{B}+\boldsymbol{b}, \rho_{0}+\rho_{1}+\rho^{\prime}, P_{0}+P_{1}+P^{\prime}$. Using the conditions (8), the linearized system (7) takes the Boussinesq form with the coefficients only depending on the radial coordinate. Hence, a normal mode expansion of the solution $F=$ $F(R) \exp (\mathrm{i}(m \phi+k z+\omega t))$ can be used with $F$ as any of the disturbed quantities.

After a normalization we find

$$
\begin{aligned}
\frac{\mathrm{d}^{2} u_{R}}{\mathrm{~d} R^{2}}+\frac{1}{R} & \frac{\mathrm{d} u_{R}}{\mathrm{~d} R}-\frac{u_{R}}{R^{2}}-\left(k^{2}+\frac{m^{2}}{R^{2}}\right) u_{R}-2 \mathrm{i} \frac{m}{R} u_{\phi} \\
- & \mathrm{i} \operatorname{Re}(\omega+m \Omega) u_{R}+2 \operatorname{Re} \Omega u_{\phi}-\frac{\mathrm{d} P^{\prime}}{\mathrm{d} R} \\
& +\mathrm{i} \frac{m}{R} \mathrm{Ha}^{2} B_{\phi} b_{R}-2 \mathrm{Ha}^{2} \frac{B_{\phi}}{R} b_{\phi}=0, \\
\frac{\mathrm{d}^{2} u_{\phi}}{\mathrm{d} R^{2}}+\frac{1}{R} & \frac{\mathrm{d} u_{\phi}}{\mathrm{d} R}-\frac{u_{\phi}}{R^{2}}-\left(k^{2}+\frac{m^{2}}{R^{2}}\right) u_{\phi}+2 \mathrm{i} \frac{m}{R} u_{R} \\
& -\mathrm{i} \operatorname{Re}(\omega+m \Omega) u_{\phi}-\mathrm{i} \frac{m}{R} P^{\prime}-\frac{\operatorname{Re}}{R} \frac{\mathrm{d}}{\mathrm{d} R}\left(R^{2} \Omega\right) u_{R} \\
& +\frac{\operatorname{Ha}^{2}}{R} \frac{\mathrm{d}}{\mathrm{d} R}\left(B_{\phi} R\right) b_{R}+\mathrm{i} \frac{m}{R} \mathrm{Ha}^{2} B_{\phi} b_{\phi}=0,
\end{aligned}
$$

$\frac{\mathrm{d}^{2} u_{z}}{\mathrm{~d} R^{2}}+\frac{1}{R} \frac{\mathrm{d} u_{z}}{\mathrm{~d} R}-\left(k^{2}+\frac{m^{2}}{R^{2}}\right) u_{z}-\mathrm{i} \operatorname{Re}(\omega+m \Omega) u_{z}$

$$
-\mathrm{i} k P^{\prime}-\operatorname{Re} \rho^{\prime}+\mathrm{i} \frac{m}{R} \mathrm{Ha}^{2} B_{\phi} b_{z}=0,
$$

$\mathrm{i}(\omega+m \Omega) \rho^{\prime}-N^{2} u_{z}=0$,

$\frac{\mathrm{d} u_{R}}{\mathrm{~d} R}+\frac{u_{R}}{R}+\mathrm{i} \frac{m}{R} u_{\phi}+\mathrm{i} k u_{z}=0$, 


$$
\begin{gathered}
\frac{\mathrm{d}^{2} b_{R}}{\mathrm{~d} R^{2}}+\frac{1}{R} \frac{\mathrm{d} b_{R}}{\mathrm{~d} R}-\frac{b_{R}}{R^{2}}-\left(k^{2}+\frac{m^{2}}{R^{2}}\right) b_{R}-2 \mathrm{i} \frac{m}{R^{2}} b_{\phi} \\
-\mathrm{i} \operatorname{Pm} \operatorname{Re}(\omega+m \Omega) b_{R}+\mathrm{i} \frac{m}{R} B_{\phi} u_{R}=0, \\
\frac{\mathrm{d}^{2} b_{\phi}}{\mathrm{d} R^{2}}+\frac{1}{R} \frac{\mathrm{d} b_{\phi}}{\mathrm{d} R}-\frac{b_{\phi}}{R^{2}}-\left(k^{2}+\frac{m^{2}}{R^{2}}\right) b_{\phi}+2 \mathrm{i} \frac{m}{R^{2}} b_{R} \\
-\mathrm{i} \operatorname{Pm} \operatorname{Re}(\omega+m \Omega) b_{\phi}+\operatorname{PmRe} R \frac{d \Omega}{\mathrm{d} R} b_{R} \\
-R \frac{\mathrm{d}}{\mathrm{d} R}\left(\frac{B_{\phi}}{R}\right) u_{R}+\mathrm{i} \frac{m}{R} B_{\phi} u_{\phi}=0, \\
\frac{\mathrm{d}^{2} b_{z}}{\mathrm{~d} R^{2}}+\frac{1}{R} \frac{\mathrm{d} b_{z}}{\mathrm{~d} R}-\left(k^{2}+\frac{m^{2}}{R^{2}}\right) b_{z} \\
-\mathrm{i} \operatorname{Pm} \operatorname{Re}(\omega+m \Omega) b_{z}+\mathrm{i} \frac{m}{R} B_{\phi} u_{z}=0,
\end{gathered}
$$

where the same symbols are used for the normalized quantities, except $P^{\prime}$, which denotes $P^{\prime} / \rho_{0}$ and redefining $\rho^{\prime}$ as $\operatorname{PmRe} g \rho^{\prime} / \rho_{0}$.

The dimensionless numbers of the problem are the magnetic Prandtl number Pm, the Hartmann number Ha, the Reynolds number Re

$$
\mathrm{Pm}=\frac{v}{\eta}, \quad \mathrm{Ha}=\frac{B_{\text {in }} R_{0}}{\sqrt{\mu_{0} \rho v \eta}}, \quad \mathrm{Re}=\frac{\Omega_{\text {in }} R_{0}^{2}}{v},
$$

and the buoyancy frequency $N$

$N^{2}=-\frac{g}{\rho_{0}} \frac{\mathrm{d} \rho_{1}}{\mathrm{~d} z}$

We used $R_{0}=\left(R_{\text {in }}\left(R_{\text {out }}-R_{\text {in }}\right)\right)^{1 / 2}$ as the unit of length, $\eta / R_{0}$ as the unit of the perturbation velocity, $B_{\text {in }}$ as the magnetic field unit, $\Omega_{\text {in }}$ as the unit of $\omega, N$, and $\Omega, R_{0} \Omega^{2}$ as the unit of $g ; \rho_{0}$ is the density unit and $v \eta / R_{0}^{2}$ is the unit of the redefined $P^{\prime}$. It is convenient to describe the influences of the density stratification and the basic rotation by the Froude number

$\operatorname{Fr}=\frac{\Omega_{\text {in }}}{N}$.

A detailed description of the numerical methods has been given in earlier papers (see e.g. Rüdiger et al. 2007b), so it will not be reproduced here. No-slip boundary conditions for the velocity on the walls are always used. The tangential electrical currents and the radial component of the magnetic field vanish on the conducting walls. For insulating walls, the magnetic field must match the external vacuum magnetic field.

\section{Hydrodynamics}

According to the Rayleigh condition, the Taylor-Couette flow with the rotation law (1) is stable for

$\mu_{\Omega}>\hat{\eta}^{2}$.

(This condition has been extended to stratified fluids by Ooyama (1966).) The general condition for nonaxisymmetric solutions is not known. It is known, however, that the axisymmetric mode is the most unstable mode for dissipative Taylor-Couette flows for $\mu_{\Omega}>0$. The nonaxisymmetric modes can be more unstable than the axisymmetric ones only for counterrotating cylinders (see Langford et al. 1988).

As we know, a stable vertical density stratification destabilizes the Taylor-Couette flow with decreasing angular velocity even beyond the Rayleigh line. This SRI is nonaxisymmetric and the most unstable mode is the mode with $m=1$.

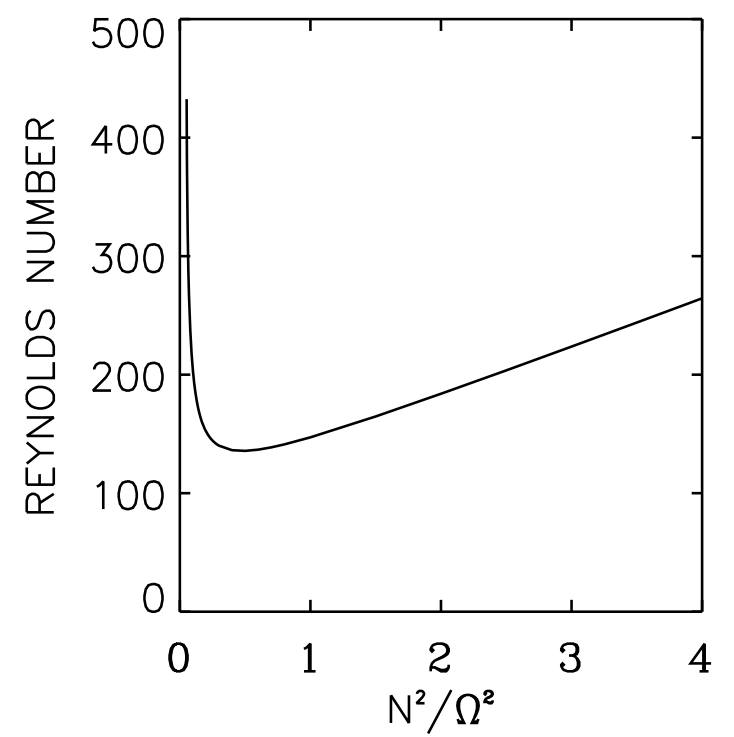

Fig. 2. The marginal-stability line for $m=1$ disturbances in a container with $\hat{\eta}=0.5$ and $\mu_{\Omega}=0.3$. The instability is stabilized for stratifications that are both too weak and too strong. The minimum Reynolds number belongs to a Froude number of 1.4 depending on the rotation law.

Shalybkov \& Rüdiger (2005) find that the instability condition is $\mu_{\Omega}<\hat{\eta}$ instead of $\mu_{\Omega}<1$, which has been confirmed experimentally by Le Bars \& Le Gal (2007). This finding, however, based on a restricted number of calculations.

In Fig. 2 the marginal-stability line (the line that separates stable and unstable regions) is calculated as a function of $N^{2} / \Omega_{\text {in }}^{2}$ for $m=1$ disturbances and for a container with $\hat{\eta}=0.5$ and with $\mu_{\Omega}=0.3$. After (14) the last value lies beyond the Rayleigh line where nonstratified flows are linearly stable.

We are looking for the minimum critical Reynolds number that exists for a buoyancy frequency $N / \Omega_{\text {in }} \simeq 0.71$, or - which is the same $-\mathrm{Fr} \simeq 1.4$. Figure 2 reveals the SRI as a delicate balance of buoyancy and centrifugal force. Both the frequencies must be approximately equal, otherwise one of the two stabilities dominates the constellation and stabilizes the flow.

The question is how this balance depends on the rotation law and on the geometry of the container. In Fig. 3 three containers are used with different gap sizes. The computations also concern various density stratifications. Three vertical lines are given for any gap size. Left is the line for the Rayleigh limit where $\Omega_{\text {out }}=\Omega_{\text {in }} R_{\text {in }}^{2} / R_{\text {out }}^{2}$. The right line mimics the rotation law $\Omega \propto 1 / R$, which is typical of galaxies $\left(\Omega_{\text {out }}=\Omega_{\text {in }} R_{\text {in }} / R_{\text {out }}\right)$, and the central line represents the Kepler rotation law $\Omega \propto R^{-1.5}$, i.e. $\mu_{\Omega}=\hat{\eta}^{1.5}$, which we call the quasi-Kepler limit. The main result of the Fig. 3 is that the quasi-Kepler rotation is always unstable. This, however, is not true for the flatter quasi-galactic rotation, which becomes stable for large gaps. From the bottom plot in Fig. 3, we have to realize that the quasi-galactic rotation law with $\Omega \propto 1 / R$ is already too flat for the possible existence of nonmagnetic SRI in galaxies.

The present calculations do not confirm the $\hat{\mu}_{\Omega}=\hat{\eta}$ line as the limit for the SRI. This limit can be higher for small-gap containers and can be lower for wide-gap containers. The stability limit seems to approach the Rayleigh line in the limit of very wide gaps and goes to unity for very narrow gaps. We ask in the following whether such results are influenced by the existence of a toroidal magnetic field. 

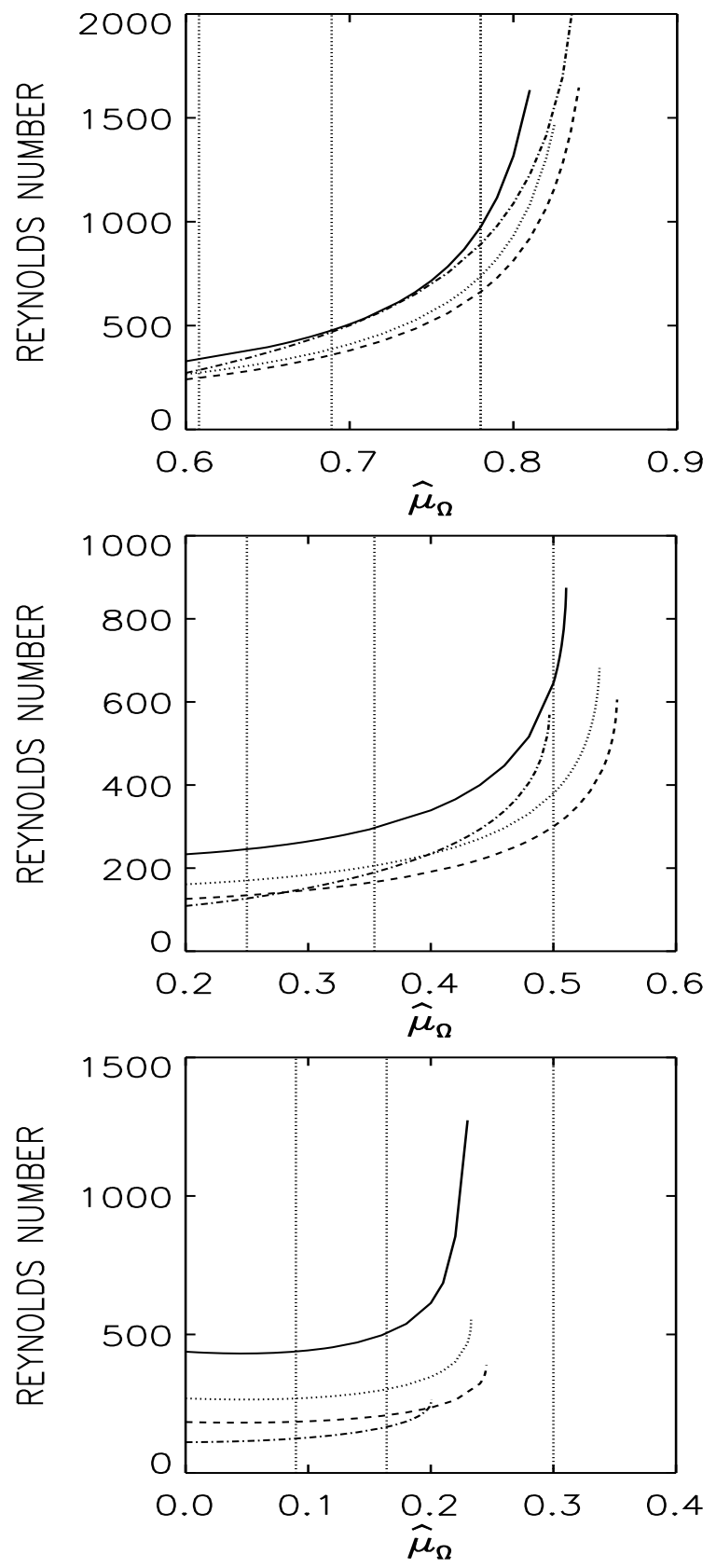

Fig. 3. The marginal-stability lines for a small gap (top, $\hat{\eta}=0.78)$, modest gap $(\hat{\eta}=0.5$, middle $)$ and wide gap $(\hat{\eta}=0.3$, bottom $)$. The vertical dotted lines denote the Rayleigh limit, the quasi-Kepler rotation, and the quasi-galactic rotation $\left(\mu_{\Omega}=\hat{\eta}\right)$. The curves represent different density stratifications with $\mathrm{Fr}=2.2$ (dot-dashed), $\mathrm{Fr}=1$ (dashed), $\mathrm{Fr}=0.7$ (dotted), $\mathrm{Fr}=0.5$ (solid, maximum stratification).

\section{SRI with azimuthal magnetic field}

Without density stratification the ideal Taylor-Couette flow with imposed azimuthal magnetic field is stable against axisymmetric disturbances, if

$$
\frac{1}{R^{3}} \frac{\mathrm{d}}{\mathrm{d} R}\left(R^{2} \Omega\right)^{2}-\frac{R}{\mu_{0} \rho} \frac{\mathrm{d}}{\mathrm{d} R}\left(\frac{B_{\phi}}{R}\right)^{2}>0 .
$$

(Michael 1954). This condition is the combination of the Rayleigh stability condition for differential rotation without magnetic field and the magnetic field stability condition without rotation. Without a global rotation, the magnetic field is stable (the second term in (15) is positive) for the magnetic field profile (4) if

$0<\mu_{B}<\frac{1}{\hat{\eta}} \equiv \hat{\mu}_{0}$

Therefore, all magnetic profiles with positive $\mu_{B}$ and with $\mu_{B}<2$ (for $\hat{\eta}=0.5$ ) are stable against axisymmetric perturbations. As the dissipative effects are stabilizing, the flow of a magnetic field with $\mu_{B}$ beyond the interval (16) becomes unstable against axisymmetric disturbances in case the magnetic field amplitude (or its Hartmann number) exceeds a critical value.

The stability condition for the azimuthal magnetic field against nonaxisymmetric disturbances after Tayler (1961) is

$\frac{\mathrm{d}}{\mathrm{d} R}\left(R B_{\phi}^{2}\right)<0$.

For the magnetic field profile (4), it takes the form

$0<\mu_{B}<\frac{4 \hat{\eta}\left(1-\hat{\eta}^{2}\right)}{3-2 \hat{\eta}^{2}-\hat{\eta}^{4}} \equiv \hat{\mu}_{1}$

for the $m=1$ (kink) mode, which is the most unstable mode. Hence, all azimuthal magnetic fields with positive $\mu_{B}$ smaller than 0.62 (for $\hat{\eta}=0.5$ ) are stable against nonaxisymmetric disturbances. Current-free fields with $B_{\phi} \propto 1 / R$, i.e. $\mu_{B}=0.5$ for $\hat{\eta}=0.5$ are thus always stable against $m=0$ and $m=1$.

As $\hat{\mu}_{1}<\hat{\mu}_{0}$, the stability interval (18) is smaller than the stability interval (16). In this sense the nonaxisymmetric ("kink") disturbances are more unstable than the axisymmetric ("sausage") disturbances. However, the situation is much more complex in the presence of (differential) rotation, and which instability really dominates depends on the parameters of the problem.

In the present paper the influence of stable vertical density stratifications on the Taylor-Couette flow stability with imposed azimuthal magnetic fields is considered for three different cases:

1) both the rotation law and the magnetic field are individually stable, and both together are unstable (i.e. $\mu_{B}=0.5$, no critical Hartmann number);

2) the magnetic field is stable for $m=0$ but unstable for $m=1$ (e.g. $\mu_{B}=1$, one critical Hartmann number);

3) the magnetic field is so steep that it is unstable for $m=0$ and for $m=1$ (e.g. $\mu_{B}=3$, two critical Hartmann numbers).

\subsection{AMRI with density stratification}

The azimuthal magnetic field with $B_{\phi} \propto 1 / R$ (i.e. $\mu_{B}=0.5$ for $\hat{\eta}=0.5)$ is considered which is current-free between the cylinders as the simplest example of an azimuthal magnetic field that is basically stable without rotation for both axisymmetric and asymmetric disturbances.

The typical stability diagram is presented by the Figs. 4 and 5 for containers with conducting/insulating cylinders, medium gap width $(\hat{\eta}=0.5$, and a rather flat rotation law (quasi-Kepler rotation with $\mu_{\Omega}=0.35$ and a slightly steeper rotation law with $\hat{\mu}_{\Omega}=0.3$ ), which are both stable without density stratification and without magnetic field. We find that the results hardly depend on the conducting properties of the cylinder material.

Figures 4 and 5 indicate the (dashed) marginal-stability line of a homogeneous fluid with $\mathrm{Pm}=1$. There are no solutions at both the vertical axis and the horizontal axis. The MHD flow is only unstable as a combination of differential rotation and the azimuthal magnetic field. For any supercritical Hartmann number, there are two critical Reynolds numbers between which the 


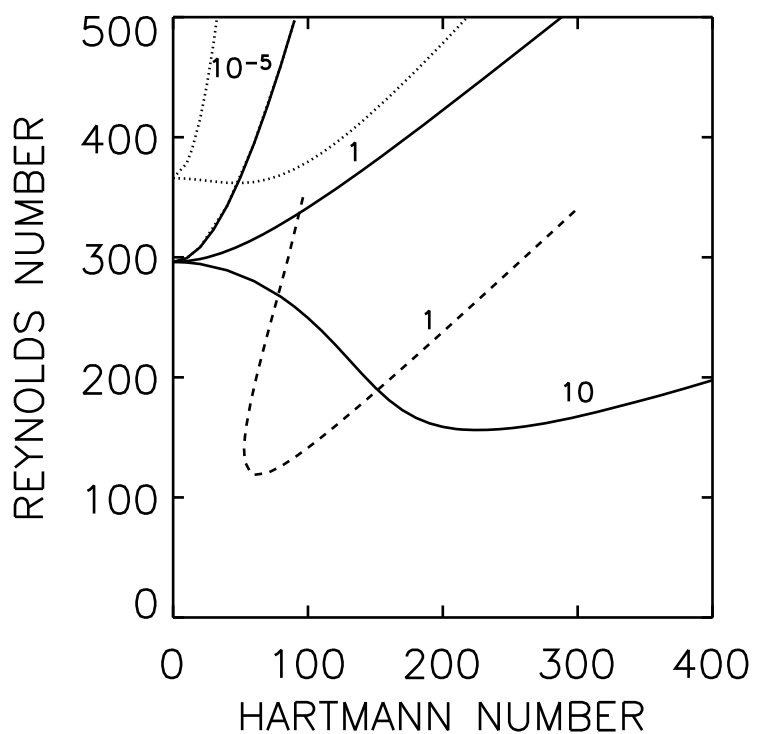

Fig. 4. The marginal-stability lines for the flow with conducting cylinders, $\hat{\eta}=0.5$, current-free $\left(\mu_{B}=0.5\right)$ azimuthal magnetic field, quasiKepler rotation $\left(\mu_{\Omega}=0.35\right), \mathrm{Fr}=0.5$ for perturbation modes with $m=1$ (solid), and $m=2$ (dotted). The curves are labeled by their magnetic Prandtl numbers. For comparison the dashed line demonstrates AMRI $(m=1)$ for homogeneous fluids $(N=0)$.

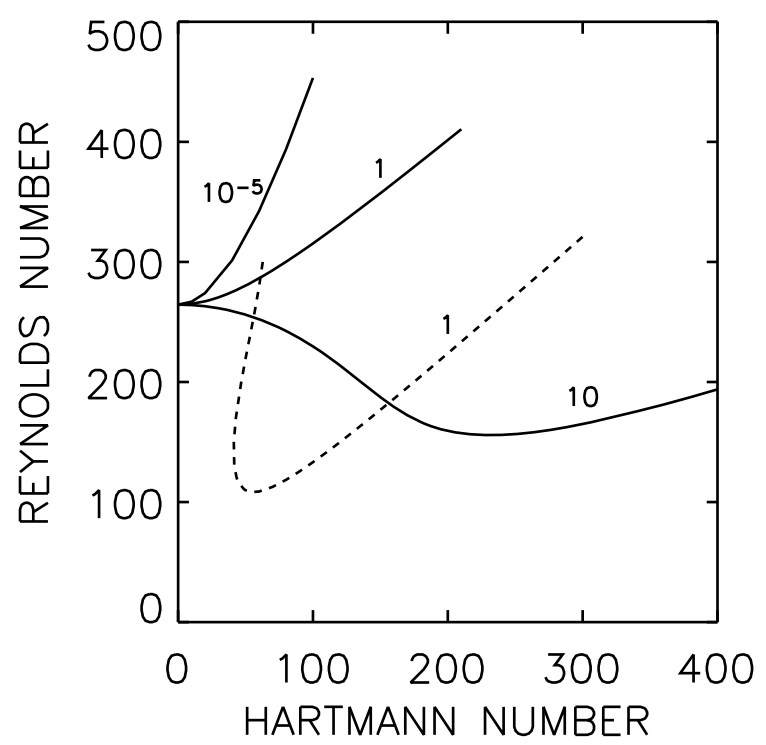

Fig. 5. The same as Fig. 4 but for insulating cylinders with $\mu_{\Omega}=0.3$ (as in Fig. 2) but without $m=2$ mode.

fluid is unstable. Similarly, for any supercritical Reynolds number, there are two critical Hartmann numbers between which the fluid is unstable. We called this phenomenon the Azimuthal MagnetoRotational Instability (AMRI), which scales with the magnetic Reynolds number in the same way as for the standard MRI in Taylor-Couette experiments. However, magnetic Reynolds numbers around 100 are too high to be realized in the MHD laboratory.

The situation changes with a density stratification $(\mathrm{Fr}=0.5)$. The following results can be interpreted as either AMRI for density-stratified fluids or as the influence of an azimuthal magnetic field on SRI. There is now an instability (SRI) without any magnetic field for $\operatorname{Re}>296$ at $m=1$ and for $\operatorname{Re}>366$ at $m=2$ (for conducting cylinders, quasi-Kepler rotation law, Fig. 4) and for $\operatorname{Re}>264$ at $m=1$ (insulating cylinders, $\mu_{\Omega}=0.3$, Fig. 5).
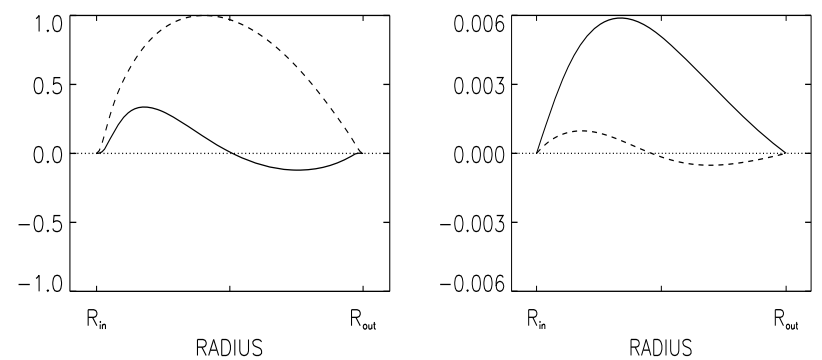

Fig. 6. The eigenfunctions for the radial components of flow $\left(u_{R}, l e f t\right)$ and field $\left(b_{R}, r i g h t\right)$ for $\mathrm{Pm}=1$. Solid: real part, dashed: imaginary part; the (arbitrary) amplitude is normalized. $\mathrm{Fr}=0.5, \mathrm{Ha}=100, m=1$, see Fig. 4.

For this case, the calculations are restricted to the kink $(m=1)$ instability.

For conducting boundaries (see Fig. 4) and for a special case $(\mathrm{Ha}=100, m=1)$ the radial eigenfunctions are given for $\mathrm{Pm}=1$ (Fig. 6), which demonstrate that the SRI modes do not form boundary phenomena. The profiles are normalized, since the amplitudes have no own physical meaning in linear theory.

For the linear growth time in units of the rotation period of the inner cylinder for the solution with $\mathrm{Pm}=1$, one obtains

$\frac{\tau_{\text {growth }}}{\tau_{\text {rot }}} \simeq \frac{243}{\operatorname{Re}-341}$.

Hence, the e-folding time is about four rotations for $\mathrm{Re}=400$. The vertical wave number at the critical Reynolds number $\mathrm{Re}=$ 341 is 8.63 , which means that the vertical cell size in units of the gap width is about 0.36 . Without magnetic field, the wave number is 8.16 . Common action of magnetic field and density stratification leads to flat cell configurations.

The influence of the azimuthal magnetic field on the SRI strongly depends on the magnetic Prandtl number Pm. For Pm $\leq$ 1 , the SRI is stabilized by the magnetic field, but it can be supported for $\mathrm{Pm}>1$ (for fixed viscosity see Figs. 4, 5). For $\mathrm{Pm}=10$ the critical Reynolds number is reduced (by a factor of two) for $\mathrm{Ha} \simeq 200$. In this case AMRI supports the SRI. This behavior is similar to that of the standard MRI with axial magnetic field and hydrodynamically unstable rotation where for $\mathrm{Pm}>1$ the magnetic field also supports the centrifugal instability (Rüdiger \& Shalybkov 2002).

Note also that the magnetic suppression of the SRI for small $\mathrm{Pm}$ is rather weak. Even for $\mathrm{Pm}=10^{-5}$, a (large) Hartmann number around 100 only leads to the small critical Reynolds number of about 500. The lines for Pm $=10^{-5}$ in Figs. 4 and 5 are identical to the lines for all smaller Pm; i.e., they also hold for gallium $\left(10^{-6}\right)$ and mercury $\left(10^{-7}\right)$. Here all the curves scale with Re rather than $\mathrm{Rm}$ so that the corresponding magnetic Reynolds numbers are quite small compared with those for AMRI. As the corresponding Hartmann numbers with Ha $\$ 100$ are also not too high, laboratory experiments with fluid metals should be possible provided a sufficiently density stratification can be produced, e.g. by heating from above (see Gellert \& Rüdiger 2008).

It is also shown in Fig. 4, that for a given Pm, the critical Reynolds numbers for $m=2$ exceed those for $m=1$. The differences, however, are not too big so that, for slightly supercritical Re, several modes should be excited. This is surprising insofar as the known smoothing action of differential rotation upon modes with high $m$ here seems to be weak.

The small Reynolds numbers and Hartmann numbers lead to the impression that the instability should be observable in the laboratory. For hydrodynamically unstable situations, we only 


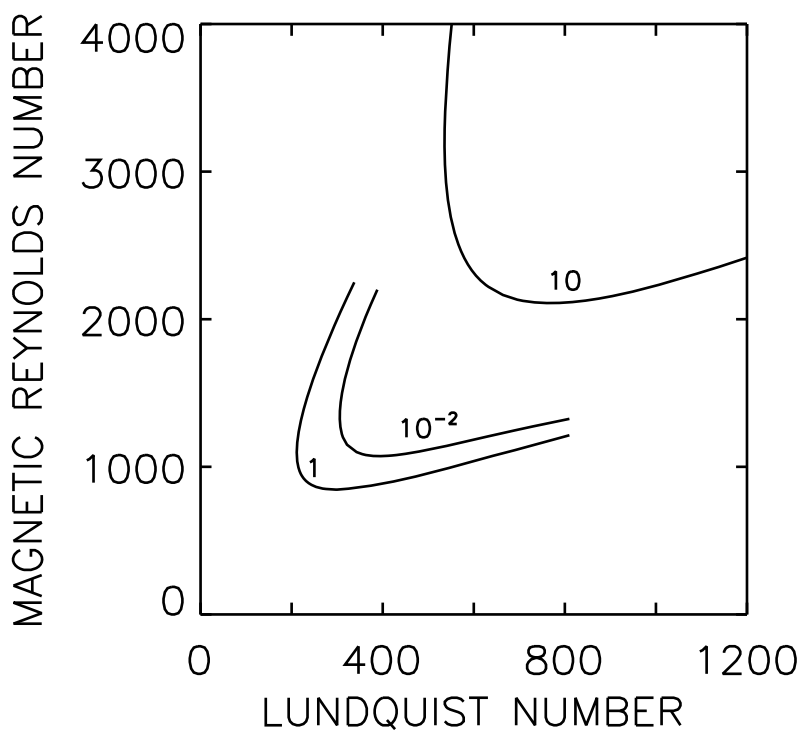

Fig. 7. AMRI for stratified fluids with $\mathrm{Fr}=0.5$ between insulating cylinders $\left(\hat{\eta}=0.5, \mu_{B}=0.5\right)$. The rotation law is so flat $\left(\mu_{\Omega}=0.6\right)$ that nonmagnetic SRI does not exist (see Fig. 3, middle). Lines are labeled by their Pm numbers.

have a suppression of the instability by the magnetic field for small Pm. For hydrodynamically stable situations (Figs. 4 and 5) it was shown for uniform fluids that the appropriate numbers of the problem are the magnetic Reynolds number Rm and the Lundquist number $S$

$\mathrm{Rm}=\mathrm{Pm} \cdot \mathrm{Re}, \quad S=\mathrm{Pm}^{1 / 2} \cdot \mathrm{Ha}$,

rather than Re and Ha. Figure 7 demonstrates that the same is true for density-stratified fluids with such flat rotation laws that hydromagnetic SRI does not operate (see Fig. 3). Both the critical magnetic Reynolds number and the Lundquist number do not vary remarkably when the magnetic Prandtl number varies over 3 orders of magnitude.

Stratified flows without magnetic field become stable for $\mu_{\Omega^{-}}$ values slightly over $\mu_{\Omega}=0.5$. According to Fig. 7 , the flow is stable for $\mu_{\Omega}=0.6$ without a magnetic field, but it becomes unstable with magnetic fields. The critical $\mathrm{Rm}$ are not monotonous with $\mathrm{Pm}$. The values of $\mathrm{Rm}$ decrease with Pm for small Pm and v.v. The result can be seen in Fig. 7, which differs in this respect from the AMRI without density stratification (see Rüdiger et al. 2007a).

The solid line in Fig. 8 demonstrates that the AMRI (like the standard MRI) exists for all rotation laws with decreasing angular velocity as a function of radius (i.e. for $\mu_{\Omega}<1$ ).

According to the Figs. 4 and 5, the marginal-stability lines have always a minimum for some Hartmann number including $\mathrm{Ha}=0$. These minimum Reynolds numbers are plotted in Fig. 8 as functions of $\mu_{\Omega}$. The dashed line is SRI without magnetic field, which disappears for rotation laws that are too flat $\left(\mu_{\Omega} \gtrsim 0.52\right)$. There is a smooth transition, however, to the instability AMRI (current-free toroidal field plus differential rotation), which has been computed here for the first time with a density gradient. The comparison with standard AMRI without $\mathrm{d} \rho / \mathrm{d} z$ yields the expected suppression by density stratification. Note, however, that for quasi-galactic rotation $\left(\mu_{\Omega}=0.5\right.$ in Fig. 8), the SRI with magnetic field can be excited more easily than without magnetic field. As the figure shows, the necessary magnetic field has a Hartmann number of about 137.

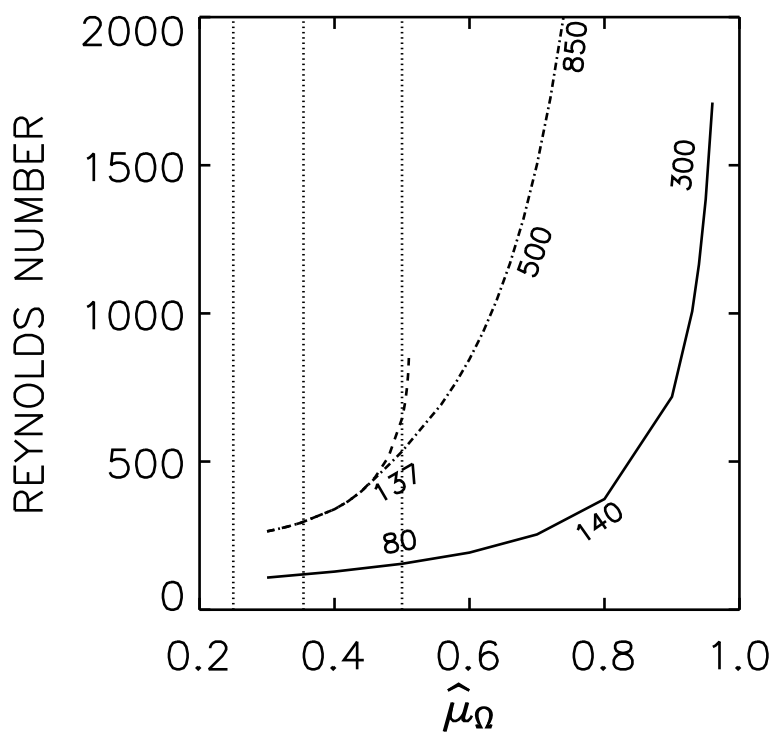

Fig. 8. The minimum Reynolds numbers for insulating cylinders, $\hat{\eta}=$ $0.5, \mathrm{Pm}=1$ and for the kink $(m=1)$ mode. The vertical lines are the same as in Fig. 3. The numbers on the curves are Hartmann numbers which correspond to the minimum Reynolds numbers. The solid line is AMRI without a stratification, the dot-dashed line is AMRI with stratification $(\mathrm{Fr}=0.5$, see Fig. 7$)$ and the dashed line is SRI without magnetic field.

Because AMRI scales with Rm rather than Re, the "smooth" transition interval from the hydrodynamic solutions to the magnetohydrodynamic scales with $\mathrm{Pm}^{-1}$. Hence, the dot-dashed line in Fig. 8 becomes steeper and steeper for decreasing Pm.

\subsection{Density stratification and Tayler instability $(m=1)$}

The influence of the vertical density stratification on the nonaxisymmetric TI (i.e. $\hat{\mu}_{1}<\mu_{B}<\hat{\mu}_{0}$ ) is considered for rotation laws that are steep enough to be Rayleigh unstable. Again the gap width is $\hat{\eta}=0.5$, the outer cylinder is resting $\left(\mu_{\Omega}=0\right)$, and the toroidal field is fairly homogeneous $\left(\mu_{B}=1\right)$ so that only the mode $m=1$ is unstable without rotation. Calculations were made for both insulating and conducting cylinders (Fig. 9). The difference between conducting and insulating boundary conditions proves to be only quantitative.

For $m=1$ the critical Hartmann numbers above which the flow is unstable for $\operatorname{Re}=0$ are 150 for conducting cylinders and 109 for insulating cylinders. These critical numbers are not influenced by the density stratification. No critical Hartmann numbers exist for $m=0$; the magnetic field stabilizes this Rayleigh-mode for both homogeneous and density-stratified fluids. For homogeneous fluids the marginal-stability line for axisymmetric disturbances does not depend on the magnetic Prandtl number Pm (Shalybkov 2006).

The critical Reynolds numbers for the Rayleigh instability for $\mathrm{Ha}=0$ do not depend on the conducting properties of the cylinders. They are $68(m=0)$ and $75(m=1)$ without stratification. The density-stratification stabilizes the flow, and the critical Reynolds numbers are 294 for $m=0$ and 226 for $m=1$ (for Fr $=0.5)$. With density-stratification, the kink mode $(m=1)$ proves to be the most unstable mode.

Moreover, for given Pm the kink mode is the most unstable one for all $\mathrm{Ha}$ for $\mathrm{Fr}=0.5$. For small Pm, this mode is stabilized by weak magnetic fields before a dramatic destabilization happens for larger magnetic fields. For increasing Pm the flow 

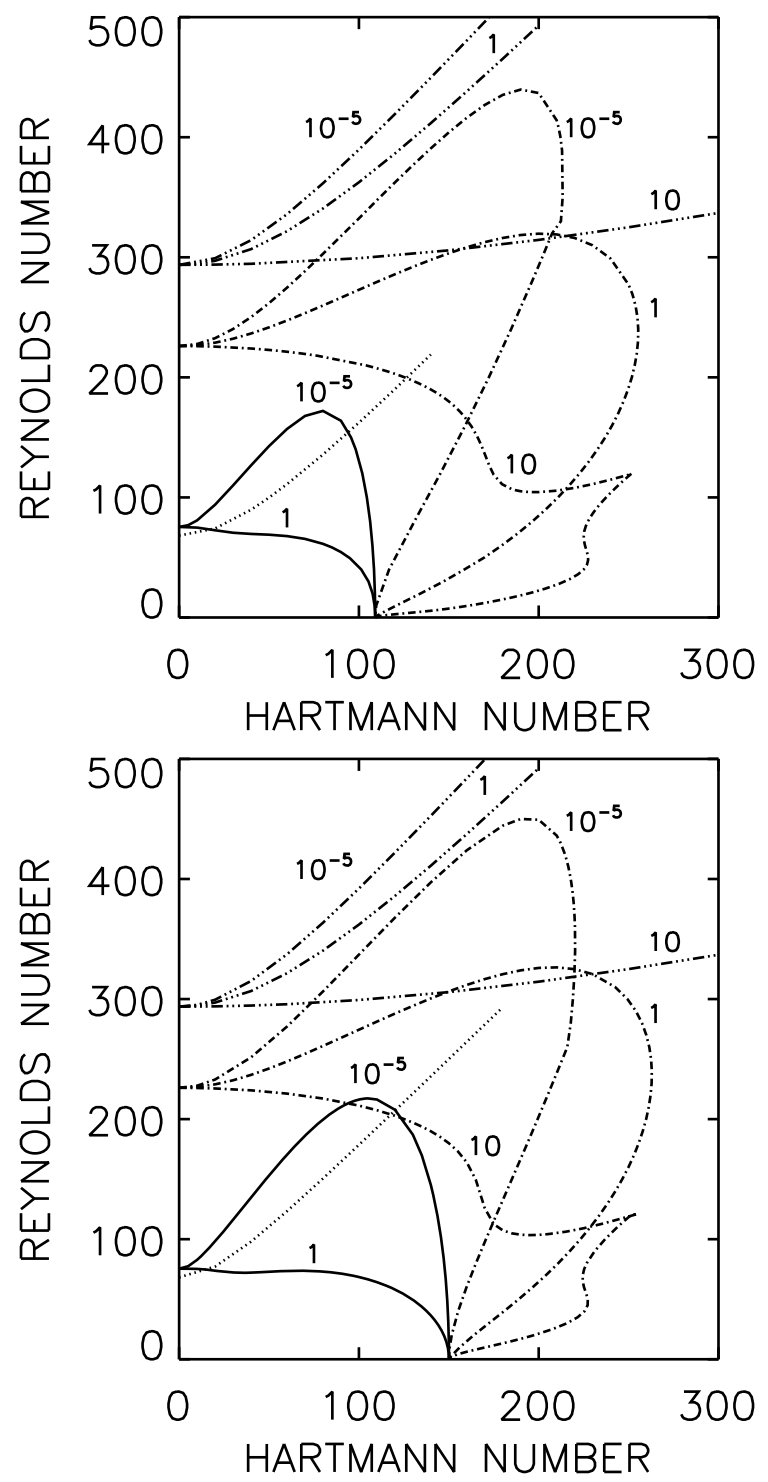

Fig. 9. The marginal-stability lines for a flow with insulating (top) and conducting (bottom) cylinders. It is $\hat{\eta}=0.5, \mu_{\Omega}=0$ (resting outer cylinder) and $\mu_{B}=1$. The curves are labeled by their magnetic Prandtl $\mathrm{Pm}$. The $m=0$ (dotted) mode (no dependence on Pm) and $m=1$ (solid) mode for homogeneous fluid and $m=0$ (dot-dot-dot-dashed) and $m=1$ (dot-dashed) modes for density-stratified fluids with $\mathrm{Fr}=0.5$.

becomes unstable for decreasing Reynolds numbers at a fixed Hartmann number. The opposite is also true: the smaller Pm, the stronger is the magnetic field influence and the flow becomes unstable for smaller Hartmann numbers at a fixed Reynolds number.

Note that the competition between magnetic and centrifugal instabilities can lead to a rather complex behavior as illustrated by the marginal-stability line with $\mathrm{Pm}=10$. Generally, however, the stability region is much larger for density-stratified fluids so that we find that a stable stratification increases the flow stability. Of particular interest is the phenomenon that, for density-stratified fluids generally, the critical Hartmann numbers are larger than without rotation and the same is true for small Pm with respect to the critical Reynolds number ("ballooning"). For large magnetic Prandtl number, however, the magnetic field basically destabilizes the Rayleigh instability. Even for weak fields, the critical Reynolds number is smaller than the Reynolds number for the nonmagnetic case.
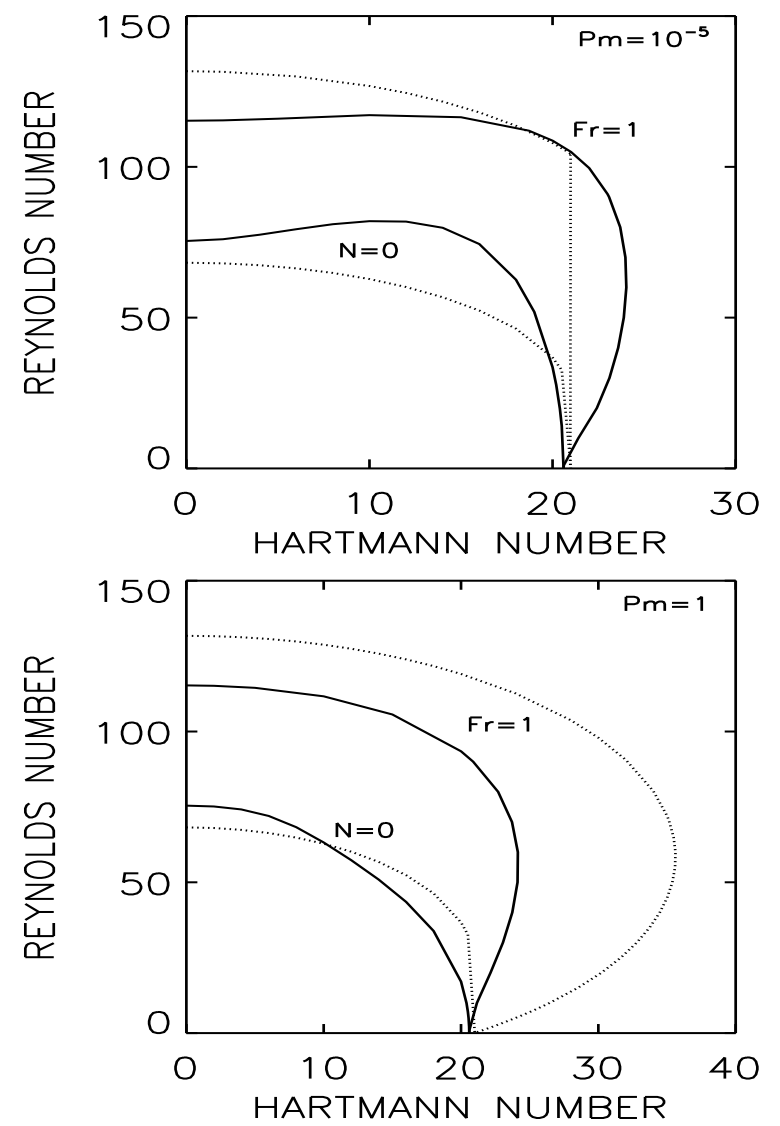

Fig. 10. The marginal-stability lines for conducting cylinders with $\hat{\eta}=$ $0.5, \mu_{\Omega}=0, \mu_{B}=3, \mathrm{Pm}=10^{-5}$ (top), and $\mathrm{Pm}=1$ (bottom). The lines connect the Rayleigh instability without magnetic field with the TI without rotation. The dotted lines are for $m=0$; the solid lines for $m=1$. The inner curves are for homogeneous fluids $(N=0)$ and the outer curves for stratified fluids with $\mathrm{Fr}=1$. The density stratification stabilizes in both directions.

\subsection{Density stratification and Tayler instability $(m=0,1)$}

The most complex situation appears when both the modes with $m=0$ and $m=1$ are Tayler-unstable. Only conducting cylinders with medium-sized gap $(\hat{\eta}=0.5)$, outer cylinder at rest and a toroidal magnetic field with strong currents $\left(\mu_{B}=3\right)$ are analyzed. The differential rotation is Rayleigh unstable without magnetic field and density stratification and the magnetic field is Tayler-unstable without rotation (for $m=0$ and $m=1$ ). The critical Hartmann number for $\mathrm{Re}=0$ is nearly equal for all cases: $m=0$ gives $\mathrm{Ha}_{0}=21$ and $m=1$ gives $\mathrm{Ha}_{1}=20.6$. For other parameters it can be more different. Either the $m=0$ or the $m=1$ mode can be the most unstable mode. Often the $m=0$ mode is also the most unstable for small Ha numbers and fast differential rotation (see Rüdiger et al. 2007b).

The critical Reynolds numbers above which the rotation becomes unstable without a magnetic field $(\mathrm{Ha}=0)$ for $m=0$ and $m=1$ are called $\operatorname{Re}_{0}$ and $\mathrm{Re}_{1}$, resp., and $\mathrm{Re}_{0}$ is smaller than $\mathrm{Re}_{1}$ for homogeneous flows with $0<\mu_{\Omega}<\hat{\eta}^{2}$. The density stratification increases $\operatorname{Re}_{0}$ and $\operatorname{Re}_{1}$ (Fig. 10). With density-stratification, the $m=1$ mode becomes the most unstable mode $\left(\operatorname{Re}_{1}<\operatorname{Re}_{0}\right)$. With a magnetic field, it is also the most unstable mode almost everywhere except for $\mathrm{Pm}=10^{-5}$ and $\mathrm{Ha}>\mathrm{Ha}_{0}$.

"Ballooning" of the stability region is produced by the density stratification. The density stratification in combination with the basic rotation stabilizes the flow for larger magnetic fields. Under the influence of differential rotation and density 

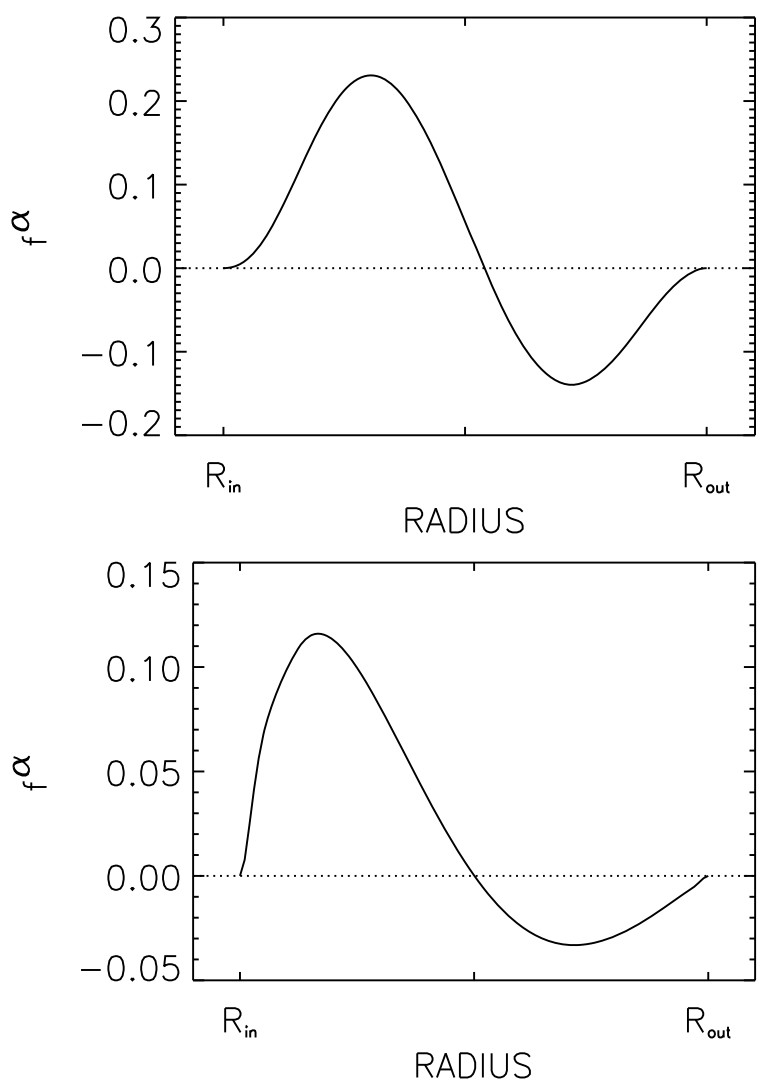

Fig. 11. The correlation function (22) for AMRI with conducting cylinders and with $\hat{\eta}=0.5, \mu_{\Omega}=0.35$ (quasi-Kepler), $\mu_{B}=0.5, \mathrm{Pm}=1$. Density stratification is zero (top) and finite (bottom, $\mathrm{Fr}=0.5$ ). The Reynolds numbers $\operatorname{Re}=141$ (top) and $\operatorname{Re}=341$ (bottom) were taken from Fig. 4.

stratification stronger magnetic field amplitudes prove to be stable than they were without rotation. The effect already exists for rather slow rotation rates (see Fig. $10, \mathrm{Pm}=1$ ). This phenomenon is a consequence of the explicit inclusion of the stable density stratification into the MHD equations. One finds that both the Rayleigh instability and the TI are stabilized by the density stratification. Without rotation, however, the density influence of the Tayler instability would remain negligibly small.

\section{Electromotive force for SRI with magnetic field, the $\alpha$-effect}

Stratorotational instability under the influence of a toroidal field is tempting to apply to the concept of the mean-field electrodynamics in turbulent fields. The nonaxisymmetric components of flow and field can be used as fluctuations, while the axisymmetric components are considered as the mean quantities. Simply the averaging procedure is the integration over the azimuth $\phi$.

It is standard to express the turbulence-induced electromotive force as

$\mathcal{E}=\left\langle\boldsymbol{u}^{\prime} \times \boldsymbol{B}^{\prime}\right\rangle=\alpha \circ\langle\boldsymbol{B}\rangle-\eta_{\mathrm{T}} \operatorname{curl}\langle\boldsymbol{B}\rangle$

with the alpha-tensor $\alpha$ and the (scalar) eddy diffusivity $\eta_{\mathrm{T}}$. In cylindric geometry, the mean magnetic field $\langle\boldsymbol{B}\rangle$ only has a $\phi$-component and the mean current $\operatorname{curl}\langle\boldsymbol{B}\rangle$ only has a $z$ component. Hence, $\mathcal{E}_{\phi}=\alpha_{\phi \phi}\left\langle B_{\phi}\right\rangle$ and $\mathcal{E}_{z}=-\eta_{\mathrm{T}} \operatorname{curl}_{z}\langle\boldsymbol{B}\rangle=0$. The latter relation only holds for AMRI where the mean magnetic field is current-free.
In the present paper we only consider the $\alpha$-effect in the frame of a linear theory where all functions are free to one and the same (complex) arbitrary parameter. This is only possible if the second-order quantities such as $\mathcal{E}_{\phi}$ are normalized with a second-order quantity. In the considered case it makes sense to form the correlation function

$f^{\alpha}=\frac{\left\langle\boldsymbol{u}^{\prime} \times \boldsymbol{B}^{\prime}\right\rangle_{\phi}}{\operatorname{MAX}\left(\sqrt{\left\langle\boldsymbol{u}^{\prime 2}\right\rangle\left\langle\boldsymbol{B}^{\prime 2}\right\rangle}\right)}$,

where all the correlations along the radius $R$ are normalized with one and the same parameter. Hence, this function no longer contains the arbitrary factor of the eigenfunctions, and by definition it is smaller than unity. The main questions are the sign of this quantity and the influence of the density stratification. Note that the $B_{\phi}$ has been given as positive in the model setup where rotation axis and density gradient are antiparallel. The latter is quite characteristic of the situation at the poles of rotating stars or disks, so that the old question of whether an $\alpha$-effect at the poles exists or not is the concern here.

One can easily show that second-order correlations of quantities running with $\exp (\mathrm{i}(k z+m \phi))$ after integration over $\phi$ do not depend on the coordinate $z$. The term $k z$ only fixes the phase where the integration starts.

The correlation function $f^{\alpha}$ can be estimated for fast-rotating magnetoconvection in the high conductivity limit $\alpha \simeq \sqrt{\left\langle\boldsymbol{u}^{\prime 2}\right\rangle}$ and with $\left\langle\boldsymbol{B}^{\prime 2}\right\rangle \simeq\left(\eta_{\mathrm{T}} / \eta\right)\langle\boldsymbol{B}\rangle^{2}$ after Krause \& Rädler (1980) so that

$f^{\alpha}<\sqrt{\frac{\eta}{\eta_{\mathrm{T}}}}$

For stellar convection, this is a small number; hence, the numerical constraints for the exact calculations of the eigenfunctions are very high. One must keep this finding in mind when discussing the numerical results given in Fig. 11 for an example without density stratification and another one with density stratification. For simplicity the magnetic Prandtl number is put to unity. Note the influence of the density stratification. The examples are taken from the Fig. 4 for $\mathrm{Ha}=100$. The resulting correlations for $N=0$ are much more antisymmetric than the values for $\mathrm{Fr}=0.5$. Averaged over the radius, the $f^{\alpha}$ vanishes for $N=0$ but not for the presence of a density stratification.

This cannot be a boundary effect. A boundary effect is implausible because the preferred radial direction is perpendicular to the rotation axis for cylinders, which mimics the equator in rotating spheres and there the $\alpha$-effect must vanish. Indeed, the antisymmetry is reduced if a density stratification is allowed (Fig. 11, bottom). Then the density gradient is now the preferred direction in the system parallel to the rotation allowing the formation of large-scale helicity (as in rotating spheres at the poles). We are thus tempted to assume that the presented calculations for the presence of a density gradient have indeed demonstrated for the existence of an $\alpha$-effect for the magnetic SRI.

Figure 12 presents similar results but for the small magnetic Prandtl number $\mathrm{Pm}=0.01$. The correlation functions provide a dominance of the positive contributions, but for higher and higher magnetic fields, the difference becomes smaller. It seems that the numerical results reflect a magnetic quenching of the $\alpha$-effect here.

\section{Conclusions}

The stability of the dissipative Taylor-Couette flow under considering the joint influence of a stable vertical density stratification 


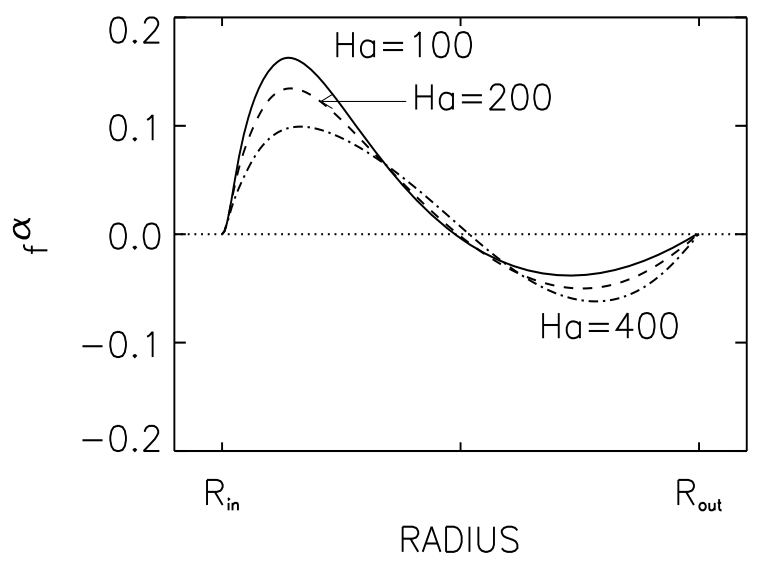

Fig. 12. The correlation function (22) for $\hat{\eta}=0.5, \mu_{\Omega}=0.35$ (quasiKepler), $\mu_{B}=0.5, \mathrm{Pm}=0.01 . \mathrm{Fr}=0.5$ ). The Reynolds numbers are $\mathrm{Re}=531$ for $\mathrm{Ha}=100$ and $\mathrm{Re}=999$ for $\mathrm{Ha}=200$ and $\mathrm{Re}=2137$ for $\mathrm{Ha}=400$. The $\alpha$-effect is quenched for stronger magnetic fields.

is considered and an azimuthal magnetic field is considered. The problem is of interest for future laboratory experiments but also within the frame of accretion disk physics. The Kepler rotation generates strong toroidal magnetic fields dominating the poloidal components. The standard MRI that works with only axial fields may not be very relevant for the stability of the Kepler rotation law compared with the azimuthal MRI in connection with the density stratification and Tayler instability. We mainly considered nonaxisymmetric "kink" modes $(m=1)$, but there are also examples where the axisymmetric modes are the most unstable ones.

We started with a discussion of the SRI without magnetic fields. For a flat rotation law, a stratification value Fr exists for which the critical Reynolds number of the rotation has a minimum (see Fig. 2). The SRI is basically stabilized both by too weak or too strong stratification. The instability only appears if the characteristic buoyancy time approaches the rotation period. Also, if the rotation is too flat, the instability disappears.

The limiting ratio $\mu_{\Omega}$ strongly depends on the gap width (see Fig. 3). For small gaps, rotation laws with $\mu_{\Omega}>\hat{\eta}$ even prove to be unstable while the condition $\mu_{\Omega}<\hat{\eta}$ results for SRI for wide gaps. Our previous finding that $\mu_{\Omega} \lesssim \hat{\eta}$ limits the SRI is only reproduced for medium-sized gaps. In all cases, however, the quasi-Kepler rotation proves to be unstable. New experiments with different gap sizes and larger Reynolds numbers could verify these results.

Figures 4 and 5 yield the basic results for SRIs subject to toroidal fields. The magnetic field is assumed to be as currentfree in the fluid between the cylinders, i.e. $B_{\phi} \propto 1 / R$ excluding TI. Without any density stratification, no Rayleigh instability exists for quasi-Kepler rotation, but nonaxisymmetric modes with $m=1$ are unstable as a result of the interaction between differential rotation and magnetic field ("AMRI").

The magnetic influence strongly depends on the magnetic Prandtl number. The instability needs a higher Reynolds number for Pm $\lesssim 1$, and it needs a lower Reynolds number for $\mathrm{Pm} \gtrsim 1$. After our experiences with MHD instabilities, this is not a surprise. It is a surprise, however, that the magnetic influence is always weak. Up to Hartmann numbers of $\mathrm{Ha} \simeq 100$, only a magnetic-induced factor of two plays a role ${ }^{2}$. Hence, our conclusion is that the SRI survives for rather high magnetic fields. For large Pm it is even supported by the toroidal magnetic field.

The combination of rotation, density stratification, and magnetic field leads to complex results. However, one basic finding is that the critical Reynolds numbers, if they exist, above which the flow becomes unstable without a magnetic field, are increased by the stable density stratification. In contrast, the critical Hartmann numbers, if any exist with the TI, above which the field becomes unstable without a rotation, do not depend on the stratification. Different transitions are possible between the two limits.

Generally speaking, the density stratification 'balloons' the stability region, and in this sense it stabilizes the flow. For slow rotation the maximal stable magnetic field exceeds the critical magnetic field without rotation while the maximal stable magnetic field is smaller for faster rotation than this critical value. Even rather low values of the Reynolds numbers lead to a stabilization of those fields that are unstable for $\mathrm{Re}=0$. The effect is strong for $\mathrm{Pm}=1$ but becomes weaker for decreasing magnetic Prandtl numbers.

For steep radial profiles of the magnetic field (i.e. strong axial currents) and magnetic Prandtl numbers Pm $\gtrsim 1$, one also finds the magnetic field destabilizing the Rayleigh instability; i.e., the critical Reynolds numbers with magnetic field are lower than the Reynolds numbers without magnetic field. This magnetic destabilization only exists for magnetic Prandtl numbers that are not too small. It exists for both uniform and densitystratified fluids (Fig. 10).

Finally, the $\phi$-component of the electromotive force representing the $\alpha$-effect of the mean-field dynamo theory has been computed. The computations require an extreme degree of accuracy. The results demonstrate the importance of the density stratification for the existence of the $\alpha$-effect. Without density stratification, the correlations are vanishing in the radial average. With an axial density stratification, the calculations model the polar region of a rotating sphere or disk. With this interpretation accepted, we found the $\alpha_{\phi \phi}$ at the northern pole as positive for negative density gradients (Fig. 11).

\section{References}

Curry, C., \& Pudritz, R. E. 1996, MNRAS, 281, 119

Dubrulle, B., Marié, L., Normand, Ch., et al. 2005, A\&A, 429, 1

Gellert, M., \& Rüdiger, G. 2008, JFM, subm.

Krause, F., \& Rädler, K.-H. 1980, Mean-field magnetohydrodynamics and dynamo theory (Oxford: Pergamon Press)

Langford, W., Tagg, R., Kostelich, E., Swinney, H., \& Golubitsky, M. 1988, Phys. Fluids, 31, 776

Le Bars, M., \& Le Gal, P. 2007, Phys. Rev. Lett., 99, 064502

Michael, D. 1954, Mathematika, 1, 45

Molemaker, M. J., McWilliams, J. C., \& Yavneh, I. 2001, Phys. Rev. Lett., 86, 5270

Ogilvie, G. I., \& Pringle, J. E. 1996, MNRAS, 279, 152

Ooyama, K. J. 1966, J. Atmos. Sci., 23, 43

Papaloizou, J. C. B., \& Terquem, C. 1997, MNRAS, 287, 771

Pitts, E., \& Tayler, R. J. 1985, MNRAS, 216, 139

Rüdiger, G., \& Shalybkov, D. 2002, Phys. Rev. E, 66, 016307

Rüdiger, G., Hollerbach, R., Schultz, M., \& Elstner, D. 2007a, MNRAS, 377 , 1481

Rüdiger, G., Schultz, M., Shalybkov, D., \& Hollerbach, R. 2007b, Phys. Rev. E, 76, 056309

Shalybkov, D. 2006, Phys. Rev. E, 73, 016302

Shalybkov, D., \& Rüdiger, G. 2005, A\&A, 438, 411

Tayler, R. J. 1961, J. Nucl. Energ. C, 3, 266

Umurhan, O. M. 2006, MNRAS, 365, 85

Yavneh, I., McWilliams, J. C., \& Molemaker, M. J. 2001, JFM, 448, 1

\footnotetext{
${ }^{2} \mathrm{Ha}=100$ for gallium corresponds to $B \approx 2200 / R_{0}$ [Gauss] with $R_{0}$ in $\mathrm{cm}$.
} 